Methods A descriptive cross sectional study was conducted in Department of Pediatrics at Darul Sehat Hospital and SESSI, Karachi from $1^{\text {st }}$ November to $3^{\text {rd }}$ February 2019.The 196 respondents were selected by convenience sampling and interview based questionnaire was administered. Variables such as age, gender, duration of illness, history of previous episodes, breastfeeding, number of vaccines, type of vaccines, reason for vaccination and not vaccinated were asked from participants.

Results A total of 196 questionnaires were interviewed from mothers of children aged 1-60 months. 98 (52.7\%) of the total was male and $88(47.3 \%)$ were females. Participants that received vaccination for rotavirus and pneumococcal vaccine were $172(88.7 \%)$ while $22(11.3 \%)$ remain unvaccinated. Incidence of watery diarrhea accounts for $66(63.5 \%)$. Frequency of diarrhoea was mild in 29(33.7\%), moderate in27 (31.4\%) and severe in $30(34.9) \%$ in vaccinated children where as it was mild in $02(11.1 \%)$ moderate in $04(22.2 \%)$ and severe in $12(66.7 \%)$ in unvaccinated children with $\mathrm{p}$ value being 0.035 . Frequency of severe pneumonia was far less then frequency of cough and cold in vaccinated participant as compared to unvaccinated participant with $\mathrm{p}$ value being $<0.001$.

Conclusion Human rotavirus and pneumococcal vaccines significantly reduced the occurrence of severe rotavirus gastroenteritis and acute respiratory tract infection. The research concludes that vaccination might not have significantly reduced the disease burden but has definitely reduced the severity of both diseases.

\section{P369 SHOULD VARICELLA VACCINATION BE INTRODUCED INTO THE NATIONAL IMMUNIZATION GUIDELINES?}

Emily Nguyen*, Olivia Baird, Mohamad Dzulkarnain, Krista Wong, Nazifa Ali-Bujang, SiewTing Ooi, Riona Kivlehan, Ciaran Power, Eleanor Molloy, Judith Meehan. Department of Paediatrics, School of Medicine, Trinity College, the University of Dublin, Dublin, Ireland

\subsection{6/archdischild-2019-epa.715}

Introduction Varicella Zoster Virus (VZV) is a member of the herpesvirus family. The virus is transmitted via respiratory droplets and presents with fever and pruritic, erythematous vesicular skin lesions. Complications to VZV although uncommon, can be severe. The virus can remain dormant in sensory ganglia with potential to reactivate as Herpes Zoster. VZV can be prevented with a live attenuated vaccine derived from the Oka strain, given over two doses 4-8 weeks apart. Currently, the vaccine is given to high risk children (immunocompromised) in the Republic of Ireland or privately for the total cost of approximately $€ 300$.

Objective To determine if the VZV vaccine should be introduced as part of the Irish National Immunisation Guidelines. Secondary aims include examining vaccine cost-effectiveness, safety, comparisons to other vaccination programs and association with Herpes Zoster.

Methods A search was performed to identify articles on varicella vaccination published between 2008-2018 using the Pubmed database. Studies which focused on specific target groups such as immunocompromised individuals, the elderly, military population, irritable bowel disease, case studies, studies were excluded.

Results Our total search identified 642 papers of which 91 were used. Implementation in Other Countries: Countries adopting universal varicella vaccine programs have shown a reduction in the incidence of varicella cases and required hospitalizations due to varicella zoster-associated complications (US, Canada, Germany, Australia). The United States implemented the program in 1996 and demonstrated a decrease of up to $89 \%$ of varicella-related cases. Safety and Side Effects:Administration of 2-dose vaccine resulted in better immunity, with no serious adverse effects. Cellulitis, anaphylaxis and breakthrough infections have been reported post-vaccine. Cost-Effectiveness and Herpes Zoster Boosting: Implementation of the vaccine would be cost-effective if considering the impact on varicella in isolation. There is no evidence to suggest a link between the VZV vaccine and an increase in Herpes Zoster.

Conclusion Implementation of the varicella vaccine appears to be cost-effective if considering the impact on varicella in isolation.

\section{P370 SEVERE NEUTROPENIA IN ADA2 DEFICIENCY}

KILIC Sara S*, Sukru Cekic, Yasin Karali. Uludag University Medical Faculty, Bursa, Turkey

\subsection{6/archdischild-2019-epa.716}

Introduction Adenosine deaminase deficiency type 2 (ADA2) is an autosomal recessive disorder caused by loss of function (LOF) mutation in ADA2 . It is characterized by early onset vasculopathy, livedo racemosa, mild immunodeficiency, systemic and CNS manifestations.. The first described monogenic vasculitis is ADA2 deficiency. Here, we described a girl with ADA2 deficiency who suffered from severe neutropenia and transient cerebral ischemic attacks.

Case A seventeen years old female patient presented with recurrent fever, lymphadenopathy, gingivitis, loss of teeth, and recurrent skin infections when she was 2 years old. Physical examination revealed multiple lymphadenomegalies on the submandibular and submental area andhepatosplenomegaly. Laboratory examination revealed neutropenia $(532 / \mu$ L) . Excisional biopsy taken from submandibular lymph node showed non-specific reactive changes in the lymph node. Bone marrow biopsy was normal except for decreased myeloid series. HAX1, ELA2 defects were excluded by Garmehausen's laboratory. G-CSF was started as 5 micrograms/ $\mathrm{kg} /$ day because of recurrent aphthous stomatitis and skin abscess. At the age of 15 , she had recurrent syncope attacks. The evaluation of cardiovascular and neurologic systems were normal. The cranial MRI was normal. A frameshift mutation (p.Arg49Alafs) was detected in CECR1 gene by kindly Raif Geha's lab. Adalimumab treatment was started $40 \mathrm{mg}$ (SC) every 2 weeks.. Her syncope attacks lost following adalimumab treatment, but neutropenia has sustained.

Discussion ADA2 deficiency is a rare and multifaced vasculitic syndrome. Neutropenia is extremely rare manifestation of this illness. Although our patient did not have livedoid skin rash, she had transient ischemic attacks of the brain suggesting cerebral vascuitis.

As a conclusion, ADA2 deficiency should be considered in cases with neutropenia, lymphoid hyperplasia and cerebral vasculitis. Adalimumab is an effective agent to prevent cerebral ischemic attacks. 